\title{
Covid-19 Peer support for junior doctors: a positive outcome of the COVID-19 pandemic?
}

\author{
Authors: Sophie Behrman, ${ }^{A}$ Nina Baruch ${ }^{B}$ and Gerti Stegen ${ }^{C}$
}

The COVID-19 pandemic has imposed new, intense and, as yet, unquantifiable strain on the wellbeing of healthcare professionals. Similarities are seen internationally with regards to the uptake of psychological support offered to healthcare professionals during a pandemic. Junior doctors are in a unique position to offer and access peer support; this is an evidence-based strategy to promote psychological wellbeing of junior doctors through the COVID-19 pandemic and into the future. The development of peer support networks during the pandemic may lead to reduced physician burnout and improved patient care in the future. We discuss a peer support initiative to support medical trainees during the COVID-19 pandemic, discuss the barriers to the success of such schemes, and reflect on the value of grass-roots peer support initiatives.

KEYWORDS: COVID-19, peer-support, junior doctors, burn-out, mental health

DOI: $10.7861 /$ fhj.2020-0069

Psychological effects of the coronavirus pandemic on junior doctors

Medical training has always taken a toll on the psychological wellbeing of doctors.' In the current pandemic, novel stressors include practical problems relating to the new ways of working required to minimise transmission of the virus, existential problems related to the disparity between individual experiences and the 'hero' narrative portrayed in the media, and the risk of moral injury ${ }^{2}$ (ie being forced into situations that violate one's moral code). Junior doctors are in a unique position. They are perhaps more accustomed to change than senior colleagues, and may be quicker to adapt to newer ways of working, but as a transitory group, they may not feel able to access emotional support from their multidisciplinary team colleagues who have well-established relationships from working together over time.

Authors: AST6 general and older adult psychiatry, Oxford Health NHS Foundation Trust, Oxford, UK; BST6 general and older adult psychiatry, Berkshire Healthcare NHS Foundation Trust, Bracknell, UK; ' Consultant psychiatrist in medical psychotherapy, Oxford Health NHS Foundation Trust, Oxford, UK

\section{Psychological effects of epidemics/pandemics on} healthcare workers

Prevalence studies from Taiwan estimate an occurrence of psychiatric morbidity in $75 \%$ of healthcare workers (HCWs) studied following the SARS epidemic, ${ }^{3}$ and in Singapore $20 \%$ of HCWs studied 2 months after the SARS epidemic had symptoms consistent with post-traumatic stress disorder. ${ }^{4}$ Although the coronavirus pandemic has posed different challenges, there is likely to be some excess psychiatric morbidity among HCWs. Just as our intensivist colleagues have learnt how best to manage COVID-19 from others abroad, we must learn from other healthcare systems how best to support our HCWs. Fostering peer support networks, and similar strategies to maintain morale and minimise isolation, have been identified as key components of minimising HCW distress during the 2003 SARS epidemic. ${ }^{4,5}$

Recent reports from Wuhan suggest that HCWs did not access formal psychological support offered, but responded well to practical concerns being addressed (eg space to rest, opportunities to communicate with their family), and were able to engage with counsellors on an ad hoc basis in their rest areas. ${ }^{6}$ Ensuring clear leadership and communication, prioritising physical safety, bolstering peer support networks, minimising isolation and normalising psychological responses are widely espoused principles that are reflected in UK guidelines for supporting the psychological wellbeing of HCWs during the pandemic.?

\section{Trainee doctors COVID-19 support group}

We have developed an online video-conferencing group for junior doctors to discuss their experiences among peers.

\section{Evolution of the group}

As a group of specialty trainees in psychiatry, we offered to facilitate peer support groups for colleagues working in acute settings through the COVID-19 pandemic. We designed a protocol for the group, informed by British Psychological Society guidance ${ }^{7}$ and following the principles of 'psychological first aid' as described by the $\mathrm{WHO},{ }^{8}$ and operated as a 'peer support' model, with trainees supporting other trainees. We have been explicit that we are not offering 'therapy' or debriefing that might risk retraumatising individuals. The style of the group was devised with input from our director of medical education, a consultant psychiatrist in medical psychotherapy. 
Initially we invited all junior doctors in the deanery to join the groups, advertising using email groups, posters and the deanery website. Uptake was slow, in keeping with experience from abroad, suggesting that formal support offered is often not adopted, particularly in the early stages of the pandemic. We wondered if poor uptake reflected doctors subconsciously employing a denial/avoidance psychological defence mechanism. While this is understandable and can be seen as an adaptive response to extraordinary stress, Greenberg and colleagues advocate being proactive in identifying colleagues who avoid discussing emotional challenges and signposting them towards support. ${ }^{2}$ Facilitating emotional avoidance may lead to adverse effects, such as reduced resilience to future stressors, mood disorder and anxiety disorders, including PTSD. ${ }^{9}$ In order to counter avoidance we worked hard to promote the group and reflected regularly on how we might enhance psychological safety and make the group more accessible.

Further into the pandemic we were approached by a junior doctor working in ICU, requesting that we run a group specifically for junior doctors in her department. While we wondered if this might be difficult for some doctors to access, especially if there were strong dynamics within the team, it became our most popular and successful group. On reflection, perhaps it is easier to achieve the psychological safety required for trainees to reflect when they are among direct peers they already know, rather than unknown colleagues they may only meet the once in the group. The ICU group participants have also expressed a feeling of experiencing shared trauma that colleagues in other specialties have not faced and, therefore, feel more comfortable attending a group only with those who have had similar experiences.

\section{Current framework of the group}

Currently we are only running a peer support group for the ICU trainees. These are run fortnightly for 45 minutes, using videoconferencing software. Groups are attended by up to six trainees and two facilitators. The groups start and close with a 'check in' and 'check out' where participants offer something about their emotional state. During the main content of the group, facilitators seek to open discussion, and use experiences of the group to normalise and validate feelings. At every group we signpost participants to other avenues of support and have escalation procedures should more direct action be required. Facilitators access supervision with other facilitators and our consultant lead following each group.

\section{Themes discussed and feedback from the groups}

Doctors attending have reported suffering from social isolation at home but also at work, with a sense that admitting to anxieties and emotions is taboo, and that seniors do not want to hear if they are struggling emotionally. Trainees have reported appreciating the opportunity to speak candidly to others to have their experiences and feelings validated, and do not feel they have time or space to do this while in their clinical settings.

Participants are directed to a brief, anonymous survey at the end of each group; from this, $100 \%$ of attendees have felt that the groups have been 'helpful'. Participants report feeling 'less lonely', 'more resilient' and 'relieved' after the groups. The experience has also been helpful for facilitators developing skills in managing groups. We seek to incorporate more formal evaluation into the design of future groups.
We have not been able to ascertain how other junior doctors are coping through the pandemic and optimistically hypothesise that many are already accessing informal peer support with direct peers through local WhatsApp groups and the like and we encourage this. However, there may be others who do not have easy access to these networks, such as doctors returning to clinical work after a break, those starting work in a new area, and doctors who are currently shielding.

\section{Conclusions}

There is certainly a role for strong leadership promoting staff psychological wellbeing during the pandemic, and evidence suggests that consulting staff about their practical priorities and addressing these, ${ }^{6}$ for example ensuring an accessible rest environment, is valuable., ${ }^{5,6}$ However, as peer support is also a key factor in maximising psychological wellbeing, there is a role for junior doctors to support each other, as is already happening organically. In England, there is the precedent of junior doctors working together across grades and specialties in the movement behind the junior doctor contract strikes in 2015; perhaps this culture of collaboration and peer support still continues.

It remains to be seen whether informal WhatsApp-based group support networks will be strong enough to sustain trainee doctors through the pandemic. It does not nullify the need for structured robust support systems for those who do not have adequate peer support, or for whom a WhatsApp chat is simply not enough. We are currently setting up other peer support groups for discrete groups of junior doctors including foundation doctors, shielding junior doctors, and Acute Care Common Stem (ACCS) trainees.

If something positive were to come of the pandemic, perhaps it will be a shift in medical culture from one of, at times, tribalism, rigid hierarchy and a denial of emotions to one which is more compassionate and accepting of emotions and where peer support is the norm. We do not wish to strip doctors of psychological defence mechanisms that may be helpful, but to add to a psychological toolbox for dealing with stress. More resilient doctors with a robust support network may be less prone to compassion fatigue and provide better patient care. There is evidence that peer support schemes have a positive impact on junior doctors' psychological wellbeing ${ }^{10}$ and reduce the risk of burnout among clinicians. A flourishing trainee doctor peer support network will hopefully promote a sense of community and collaboration and provide meaning to experiences, which reduce burnout and ultimately improve patient care. ${ }^{11}$

\section{Acknowledgements}

We would like to thank our co-facilitators in the HETV Covid Peer Support Group and members of the Trainees Advisory Committee who have helped set up and publicise the group.

\section{References}

1 British Medical Association. Caring for the mental health of the medical workforce. BMA, 2019. www.bma.org.uk/media/1365/ bma-caring-for-the-mental-health-survey-oct-2019.pdf.

2 Greenberg N, Docherty M, Gnanapragasam S, Wessely S. Managing mental health challenges faced by healthcare workers during covid-19 pandemic. BMJ 2020;368:m1211.

3 Chong M-Y, Wang W-C, Hsieh W-C et al. Psychological impact of severe acute respiatory syndrome on health workers in a tertiary hospital. Br J Psychiatry 2004;185:127-33. 
4 Chan OA, Huak CY. Psychological impact of the 2003 severe acute respiratory syndrome outbreak on healthcare workers in a medium size regional general hospital in Singapore. Occ Med 2004;54:190-6.

5 Maunder R, Hunter J, Vincent L et al. The immediate psychological and occupational impact of the 2003 SARS outbreak in a teaching hospital. Can Med Assoc J 2003;168:1245-51.

6 Chen Q, Liang M, Li Y et al. Mental health care for medical staff in China during the COVID-19 outbreak. Lancet Psychiatry 2020; 7:e15-16.

7 British Psychological Society Covid19 Staff Wellbeing Group. Guidance: The psychological needs of healthcare staff as a result of the Coronavirus pandemic. BPS, 2020. Available from www.bps.org.uk/ news-and-policy/psychological-needs-healthcare-staff-resultcoronavirus-pandemic.

8 World Health Organization. Psychological first aid: guide for field workers. WHO, 2011. Available from www.who.int/mental_health/ publications/guide_field_workers/en/.
9 Salters-Pedneault K, Tull MT, Roemer L. The role of avoidance of emotional material in the anxiety disorders. Appl Prevent Psychology 2004;11:95-114.

10 Chanchlani S, Chang D, Ong JS, Anwar A. The value of peer mentoring for the psychosocial wellbeing of junior doctors: a randomised controlled study. Med J Aust 2018;209:401-5.

11 West CP, Dyrbye LN, Shanafelt TD. Physician burnout: contributors, consequences and solutions. J Intern Med 2018;283:516-29.

Address for correspondence: Dr Sophie Behrman, Vaughan Thomas Ward, Warneford Hospital, Warneford Lane, Headington, Oxford OX3 7JX, UK.

Email: sophie.behrman@oxfordhealth.nhs.uk 